\title{
DISTRIBUCIÓN GEOGRÁFICA Y PLANTAS SUSTRATO DE SEMIDALIS KOLBEI ENDERLEIN, 1906 (NEUROPTERA: CONIOPTERYGIDAE) EN LA REGIÓN DE LA ARAUCANÍA, CHILE
}

\author{
GEOGRAPHIC DISTRIBUTION AND SUBSTRATE PLANTS OF SEMIDALIS \\ KOLBEI ENDERLEIN, 1906 (NEUROPTERA: CONIOPTERYGIDAE) \\ IN THE REGION OF LA ARAUCANÍA, CHILE
}

Ramón Rebolledo R. ${ }^{1}$; Pamela Pinot B. ${ }^{1}$; Nelson Ojeda O. ${ }^{1}$; Alfonso Aguilera P. ${ }^{1}$

\section{RESUMEN}

Semidalis kolbei es uno de los más conocidos de la familia Coniopterygidae chilenos. Sin embargo, aun persisten aspectos desconocidos de su biología tales como: distribución por región, hospederos, sustratos vegetales, ciclo estacional y vital y menos todavía para la región de La Araucanía. Razón por la cual en el presente trabajo se entregan aspectos de su distribución en la región, así como los sustratos vegetales en donde es posible encontrarlo. Para determinar las especies vegetales donde se encuentra $S$. kolbei se muestrearon las cinco áreas agroecológicas de Rouanet (1988), en las cuales se realizaron barridos de red entomológica en especies vegetales como bosque nativo, plantaciones forestales entre otras. Para determinar la distribución de $S$. kolbei se utilizó el sistema de posicionamiento global (GPS) y para construir los mapas de distribución se utilizó el sistema de posición geográfica con el programa Arc View Gis 3.2. Los resultados muestran que S. kolbei está ampliamente distribuido en la Región, siendo además una especie abundante, la que es posible encontrarla desde el nivel del mar hasta los 1.624 m.s.n.m en árboles como coigüe, araucaria y lenga.

Palabras clave. Semidalis kolbei, distribución, abundancia, sustratos vegetales.

\begin{abstract}
Semidalis kolbei is one of the most known Coniopterygidae pertaining to vegetal Chilean family. However, unknown aspects of its biology still persist, such as: distribution by region, host, vegetal substrates, life and seasonal cycle and much less for the region of La Araucania. For that reason in the present assay aspects of its distribution in the region are given, as well as the vegetal substrates in where it is possible to find it. In order to determine the vegetal species where S. kolbei five Rouanet's agro ecological areas (1988) were sampled. In them, sweeping with an entomological net in vegetal species as native forest was made, forest plantations among others. To determine S. kolbei distribution, systems of global positioning (GPS) were used and the distribution maps made, a system of geographic position with Arc View Gis 3.2 program was used. The results show that $\mathrm{S}$. kolbei is widely distributed in the Region being, besides an abundant species, which is possible to be found from the sea level to 1.624 MASL in trees such as coigüe, araucaria and lenga.
\end{abstract}

Key words: Semidalis kolbei, distribution, abundance and vegetal substrates.

\section{INTRODUCCIÓN}

Los representantes de la familia Coniopterygidae son los neurópteros más pequeños del grupo, con una longitud de alas anteriores de 2 a $4 \mathrm{~mm}$; super- ficialmente semejan moscas-blancas (Hemiptera; Aleyrodidae), pero se diferencian por la postura característica de sus alas en forma de techo cuando están en reposo (Artigas, 1994). Esta familia presenta una amplia distribución mundial, con un alto

$1 \quad$ Facultad de Ciencias Agropecuarias y Forestales, Universidad de La Frontera, Casilla 54-D. Temuco, Chile. E mail: ramonr@ufro.cl

Fecha de Recepción: 09 Mayo 2007

Fecha de Aceptación: 14 Septiembre 2007 
número de especies (aproximadamente 450), siendo algunas de ellas importantes en el control natural y biológico de algunas plagas de la agricultura (New, 1989, 2001, Monserrat y Bayo, 1995; Monserrat, 2005; Richards y Davies, 1983).

Son insectos extremadamente frágiles, teniendo un parecido general con los áfidos. Pueden ser separados fácilmente de las otras familias del orden Neuroptera por su tamaño pequeño, su venación reducida sin ramificaciones terminales y la presencia de secreción cerosa en las alas y el cuerpo (Artigas, 1994, Chinery, 1998). Las alas son grises oscuras, empolvadas finamente con cera. Por lo general no tienen manchas, pero algunas especies pueden tener un patrón de puntos blancos. La cabeza y el tórax son negros con el abdomen café pálido. Los machos pueden ser distinguidos de las hembras por el extremo negro del abdomen de éstos (Barbagallo, 1991; Costa et al., 1994).

Los adultos son activos durante la noche y son atraídos por la luz. Durante el día reposan sobre el follaje de árboles y arbustos desde los cuales ellos pueden volar si son molestados (Costa et al., 1991). De apariencia frágil, con el cuerpo y alas cubiertas de una exudación pulverulenta blanquecina. (Borror et al., 1992; Costa et al., 1991; Gillot, 1995).

Muchas de las especies de los bosques viven en las copas altas de los árboles. Algunas especies son aparentemente específicas a ciertas especies de plantas. Se alimentan de una dieta mezclada de insectos pequeños y excreciones dulces producidas por homópteros. Los huevos son puestos solos o en pequeños grupos sobre las hojas o la corteza. Los diminutos coniopterígidos son activos depredadores de insectos, escamas y sobre todo ácaros que se encuentran en los árboles (Costa et al., 1991; Gillot, 1995).

Las larvas son activas depredadoras y se caracterizan por sus mandíbulas pequeñas y palpos grandes en forma de saco. Hilan sus capullos en el follaje o la corteza. Debido a su predilección por insectos, escamas y ácaros, los coniopterígidos han sido usados como agentes de control biológico (Lloréns, 1990 a, b; Lloréns y Garrido, 1992).

En Chile se conocen pocas especies de coniopterígidos, la mayoría de ellas se ha registrado en la zona central del país. La especie más frecuente en Chile es Semidalis kolbei Enderlein, de la cual se han encontrado representantes desde la Región de Coquimbo (Ñague, Los Vilos) hasta el norte de la Región de Magallanes y Antártica (Puerto Natales) y desde la cordillera de los Andes hasta la costa del Pacífico (Adams, 1973). El mismo autor indica que S. kolbei es muy abundante en todo Chile y que ha sido reportada desde la IV Región de Coquimbo hasta la XII Región de Magallanes y desde la costa Pacífico hasta altitudes de $1.350 \mathrm{msnm}$ en la Cordillera de los Andes. A lo anterior Monserrat (2005) señala que $S$. kolbei está presente también en las provincias de Neuquén, Río Negro y Chubut de la República Argentina.

Monserrat (2005) realiza un importante aporte con respecto a los sustratos vegetales, donde es posible encontrar ejemplares de $S$. kolbei, entregando una detallada lista de 22 especies de plantas, más la altura sobre el nivel del mar donde encontrase registro de esta especie.

A pesar de ser $S$. kolbei la especie mejor conocida de los coniopterígidos chilenos, todavía se desconocen importantes aspectos de su biología, tales como ciclo vital, estacional y hábitos de consumo. En este último aspecto, Prado (1991) la cita como depredadora del ácaro Oligonychus yothersi.

No obstante lo anterior, la Región de La Araucanía (Chile) no fue muestreada por Monserrat (2005). Por lo que no existen antecedentes de la presencia de $S$. kolbei, ni menos sobre aspectos de distribución geográfica y sustratos vegetales donde es posible encontrarlo pensando en futuros programas de control biológico. Por lo tanto este trabajo tiene como objetivo general estudiar la presencia de S. kolbei en la Región de La Araucanía y como objetivos específicos a) determinar los sustratos vegetales donde se encuentra S. kolbei y b) elaborar mapas de distribución geográfica basada en Sistemas de Posicionamiento Global (GPS) y Sistemas de Información Geográfica (GIS).

\section{MATERIALES Y MÉTODOS}

El trabajo de terreno se llevó a cabo en las cinco zonas agroecológicas de la Región de La Araucanía propuestas por Rouanet et al. (1988) y también en la distribución territorial de la vegetación natural desarrollada por Gajardo (1994). Se establecieron en cada una de estas zonas estaciones de observación y muestreo, de acuerdo a las características vegetacionales del área de estudio. Las capturas se realizaron en distintas fechas durante la primaveraverano de los años 2003-2004 y 2004-2005, con una periodicidad de 15 días. 
Los ejemplares para el estudio fueron colectados en distintos lugares: Cholchol, Curarrehue, Curacautín, Queule, Lago Budi, Laguna Malleco, Lonquimay, Vilcún, Trovolhue, Ercilla, Lastarria, Loncoche, Puerto Saavedra, Collipulli y Angol. Estos lugares se georreferenciaron, y los puntos registrados se promediaron. En cada uno de estos lugares se muestreó de 10:00 a 18:00 horas. Dependiendo de los sectores, se establecieron como mínimo seis estaciones debidamente georreferenciadas (coordenadas UTM y altitud). En cada estación de muestreo se realizaron barridos de red entomológica a toda la vegetación existente en cada lugar (hierbas, arbustos y árboles). En el caso particular de los árboles la red abarcaba ramas entre 1 a 3 metros. Una vez recolectados con la red entomológica, los ejemplares eran trasladados vivos al laboratorio de Entomología Aplicada de la Universidad de La Frontera, en frascos plásticos de $8,8 \mathrm{~cm}$ de diámetro interior, $11.5 \mathrm{~cm}$ de diámetro superior y $8 \mathrm{~cm}$ de alto, con tapa previamente perforada para permitir el paso del aire al interior del mismo.

Para la realización de los mapas de distribución geográfica se utilizó el programa computacional Arcview GIS versión 3.2. (Bosque, 1992; Felicisimo et al., 1994; Penín, 2002).

Los resultados fueron sometidos a un análisis estadístico de la prueba de correlación no paramétrica Rho de Spearman (nivel de significancia 0,01) (Visauta, 1997, 1998).

\section{RESULTADOS Y DISCUSIÓN}

Semidalis kolbei se distribuye en todas las localidades de las cinco zonas agroecológicas (Figura 1) de la Región.

La mayor cantidad de S. kolbei fue encontrada en las cercanías de Lonquimay con 229 ejemplares, seguido de la Laguna Malleco donde la captura

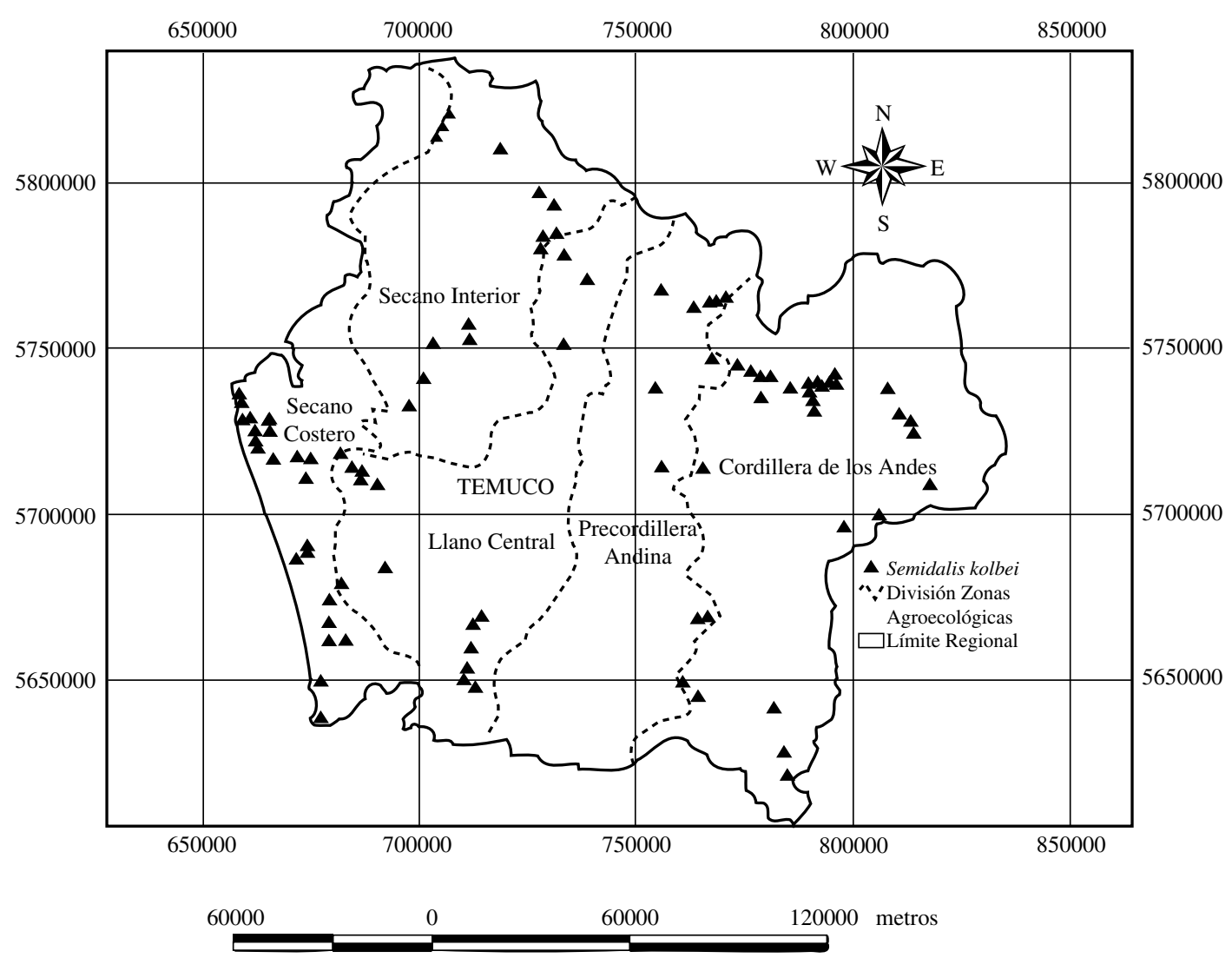

Figura 1. Mapa de distribución de Semidalis kolbei en las zonas agroecológicas de la Región de La Araucanía, temporadas 2003-04 y $2004-05$. 
alcanzó los 58 ejemplares. Curacautín con 48 ejemplares. En el predio Santa Violeta ubicado en la comuna de Vilcún se recolectaron 31 insectos. En las localidades de Angol y Cholchol se encontraron 19 ejemplares. En la localidad de Trovolhue, comuna de Puerto Saavedra, se recolectaron 15 individuos. Cada uno de los ejemplares de S. kolbei fue encontrado en diferentes sustratos vegetales de las comunidades arbóreas muestreadas (Cuadro 1). En cambio, la localidad de Curarrehue y en las cercanías de Queule fueron los lugares donde se capturaron menos ejemplares de este insecto. Sin embargo, en la localidad de Curarrehue el muestreo se realizó a mediados de marzo, razón por la cual se capturaron pocos insectos. Distinto fue el caso de Queule, allí los muestreos fueron realizados a fines de diciembre y donde la vegetación era mayoritariamente Pinus radiata.

En el Cuadro 1 se muestran los sustratos vegetales donde fue registrado $S$. kolbei. Es necesario destacar que no se encontraron ejemplares de $S$. kolbei en el estrato herbáceo y que faltó analizar árboles frutales, donde probablemente el insecto se encuentre presente. Estos resultados coinciden con lo registrado por Monserrat (2005) quien da una amplia lista de árboles y arbustos en los cuales es posible encontrar ejemplares del insecto. Los nuevos registros de este trabajo corresponden a roble, boldo, pillopillo, avellano, ñirre, maitén, canelo, peumo. Sin embargo, a diferencia de Monserrat (2005), en el presente estudio no se encontraron ejemplares de $S$. kolbei en Pinus radiata D. Don (pino insigne).

La mayor cantidad de insectos fueron encontrados en Araucaria araucana (202 ejemplares), Nothofagus obliqua (67 ejemplares), Nothofagus dombeyi (53 insectos), Nothofagus pumilio (47 ejemplares), Реити boldus (135 ejemplares), Cryptocarya alba (122 ejemplares) y Aristotelia chilensis (128 ejemplares).

Cuadro 1

Presencia de Semidalis kolbei en distintos sustratos vegetales, temporadas 2003-04 y 2004-05

\begin{tabular}{|c|c|c|}
\hline Nombre vulgar & Nombre científico & $\mathbf{N}^{0}$ ejemplares de Semidalis kolbe \\
\hline Roble & Nothofagus obliqua (Mirb.) Oerst. & 67 \\
\hline Quila & Chusquea quila Kunth. & 34 \\
\hline Nirre & Nothofagus antarctica (G. Forster) Oerst & 9 \\
\hline Araucaria & Araucaria araucana (Mol.) Koch. & 202 \\
\hline Raulí & Nothofagus nervosa (Poepp. et Endl.) & 23 \\
\hline Coigüe & Nothofagus dombeyi (Mirb.) & 53 \\
\hline Arrayán & Luma apiculata (DC.) Burret & 19 \\
\hline Maitén & Maytenus boaria Mol. & 15 \\
\hline Peumo & Cryptocarya alba (Mol.) Looser & 122 \\
\hline Canelo & Drimys winteri J.R. Forst y G. Forst & 15 \\
\hline Lenga & Nothofagus pumilio (Poepp. et Endl.) & 47 \\
\hline Boldo & Peumus boldus Mol. & 135 \\
\hline Radal & Lomatia hirsuta (Lam.) Diles. Ex Macbr. & 15 \\
\hline Aromo & Acacia melanoxylon (L.) Willd. & 8 \\
\hline Pillopillo & Ovidia pillopillo (Gay) Meisn & 5 \\
\hline Lleuque & Prumnopitys andina (Poepp \& Endl.) De Lanb. & 5 \\
\hline Maqui & Aristotelia chilensis (Mol.) Stuntz & 128 \\
\hline
\end{tabular}


Con respecto a la altitud (Cuadro 2), S. kolbei se encontró en todos los lugares de muestreo y a diferentes niveles de altitudes que fueron desde los 16 m.s.n.m. en Puerto Saavedra hasta los 1.624 m.s.n.m. en Lonquimay. Esto amplía el rango descrito por Monserrat (2005), quien señala la presencia de esta especie en un rango de alturas entre $\operatorname{los} 10 \mathrm{y}$ 800 m.s.n.m.

Tomando en cuenta lo observado en el análisis del coeficiente de correlación de Rho Spearman, entre altitudes y el número de ejemplares colectados, éstos mostraron un coeficiente de correlación positiva ( $\mathrm{R}$ de 0,385 ). Por lo tanto, existe una relación entre las altitudes de los muestreos y la cantidad de ejemplares de Semidalis kolbei encontrados.

En relación a la distribución geográfica de S. kolbei en la Región de La Araucanía (Figura 1), ésta se registró en las cinco zonas agroecológicas descritas por Rouanet et al. (1988). En la Figura 1 se observa el mapa con la totalidad de puntos donde

\section{Cuadro 2}

Altitud máxima de las diferentes localidades muestreadas, temporadas 2003-04 y 2004-05

\begin{tabular}{|l|c|}
\hline \multicolumn{1}{|c|}{ Localidad } & Altitud (m.s.n.m.) \\
\hline Cholchol & 129 \\
\hline Curacautín & 831 \\
\hline Queule & 49 \\
\hline Collipulli & 377 \\
\hline Angol & 658 \\
\hline Lago Budi & 25 \\
\hline Laguna Malleco & 670 \\
\hline Lonquimay & 1624 \\
\hline Vilcún & 710 \\
\hline Curarrehue & 498 \\
\hline Trovolhue & 51 \\
\hline Lastarria & 476 \\
\hline Loncoche & 106 \\
\hline Lago Colico & 106 \\
\hline Collipulli & 235 \\
\hline Puerto Saavedra & 16 \\
\hline Nehuentué & 390 \\
\hline
\end{tabular}

se realizaron los registros, el cual muestra una gran distribución en la Región, ya que en casi todos los lugares muestreados se registraron ejemplares. Una característica encontrada en la distribución geográfica de $S$. kolbei fue la mayor abundancia relativa en árboles de hoja perenne, como Araucaria araucana y Peumus boldus.

\section{CONCLUSIONES}

- Semidalis kolbei está presente en las cinco zonas agroecológicas de La Araucanía.

- Respecto a la altitud donde se realizaron los muestreos, se dio una correlación positiva entre los ejemplares capturados y la altitud donde se realizaron los registros. La mayor cantidad de ejemplares colectados se encontró en las zonas de mayor altitud.

- El sustrato con mayor presencia de Semidalis kolbei fue Araucaria araucana (Mol.) Koch.

- Semidalis kolbei tuvo una mayor presencia en los sustratos vegetales de hoja perenne.

\section{AGRADECIMIENTOS}

Este estudio contó con financiamiento del Proyecto DIDUFRO 120303

\section{LITERATURA CITADA}

ADAMS, P. 1973. Coniopterygidae from Chile, with three New Species (Neuroptera). The Pan-Pacific Entomologist 49:250-254.

ARTIGAS, J. 1994. Entomología económica: insectos de interés agrícola, forestal, médico y veterinario (Nativos, introducidos y susceptibles de ser introducidos). Vol. I. Ediciones Universidad de Concepción. Concepción, Chile. 1126 p.

BARBAGALLO, S. 1991. Gli afidi: organizzazione, comportamenti, bio-ecologici, rapporti con le piante ospiti In S. Barbagallo Gli afidi e le colture agrarie. Reda Edizione per 1'agricoltura. Roma. Pp. 17-49

BORROR, D.; CH. TRIPLEHORN, Y N. JOHNSON. 1992. An introduction to me study of insects. Saunders College Publishing. New York 875 p.

BOSQUE, J. 1992. Sistema de Información Geográfica. Ediciones Rialp. SA. I Edición. Madrid. 451 p.

CHINERY, M. 1988. Insectos de España y Europa. Edición Omega. Barcelona, España. 4002 p.

COSTA, J; F. FERRAGUT; F. GARCÍA Y J. M. LLORÉNS. 1991. Ácaros de las plantas cultivadas y su control biológico. Pisa Ediciones. Valencia, España. 175 p. 
COSTA, J; F. FERRAGUT Y F. GARCÍA. 1994. Plagas agrícolas, Agropubli. Phytoma España. Valencia, España. $376 \mathrm{p}$.

FELICISIMO, A.; E. FRANCES; J. FERNÁNDEZ; A. GONZÁLEZ-DIEZ, \& J. VARAS 1994. Modeling the potential distribution of forest with a GIS. Photogrammetric Engineering and remote sensing 68(5):455-461.

GAJARDO, R. 1994. La vegetación natural de Chile. Clasificación y distribución geográfica. Editorial Universitaria. Santiago, Chile.165 p.

GILLOT, C. 1995. Entomology. Plenum Press. Second edition. New York. 729 p.

HOFFMANN, A. 1978. Flora Silvestre de Chile Zona Central. Ediciones Fundación Claudio Gay. Santiago, Chile. $255 \mathrm{p}$.

LLORÉNS, J. 1990a. Homoptera I. Cochinillas de los cítricos y su control biológico. Pisa Ediciones. Valencia, España. 260 p.

LLORÉNS, J. 1990b. Homoptera II: pulgones de los cítricos y su control biológico. Pisa Ediciones. Valencia, España. $170 \mathrm{p}$.

LLORÉNS, J. Y A. GARRIDO. 1992. Homoptera III. Mosca blanca y su control biológico. Pisa Ediciones. Valencia, España. 203 p.

NEW, T. 1989. Planipennia, Lacewings. In Walter de Greyter (ed.) Handbuch der Zoologie. Vol 4. pp. 129.

NEW, T. 2001. Introduction to the systematic and distribution on the Coniopterygidae, Hemerobiidae and Chrysopidae used in pest management. In McEwen, P.; T. New \& A.
Whittington (eds.) Lacewings in the Crop Environment. Cambridge. Uni. Press:6-28.

MONSERRAT, V. y J. BAYO. 1995. Nuevos datos sobre los coniopterígidos de México central (Neuroptera: Coniopterygidae). Boln. Asoc. esp. Ent., 19 (3-4): 125-130.

MONSERRAT, V. 2005. New dat of th dusty wings from Coquimbo, Patagonia and Tierra del Fuego (Neuroptera, Coniopterygidae). Entomofauna Zeitschrift Für Entomologie 25:421-436.

PENÍN, M. 2002. Desarrollo de un sistema gestor de rutas de posicionamiento global por satélite. Facultad de informática. Universidad de La Coruña. La Coruña, España. 108 p.

PRADO, E. 1991. Artrópodos y sus enemigos naturales asociados a las plantas cultivadas en Chile. Boletín técnico $\mathrm{N}^{\circ} 169$. INIA La Platina. Santiago, Chile. 207 p.

RICHARDS, O. \& R. DAVIES. 1983. Tratado de entomología Imms: Clasificación y biología. Tomo II. Editorial Omega. Barcelona, España. 998 p.

ROUANET, J.; O. ROMERO; y R. DEMANET. 1988. Áreas Agroecológicas en la IX Región. Descripción. Investigación y Proceso Agropecuario, Carillanca, INIA (Temuco, Chile)7(1):18-23.

VISAUTA, B. 1997. Análisis estadístico con SPSS para Windows. Estadística Básica. Ed. McGraw-Hill/Interamericana de España. Madrid. 304 p.

VISAUTA, B. 1998. Análisis estadístico con SPSS para Windows. Estadística multivariante. Ed. McGraw-Hill/Interamericana de España. Madrid. 304 p. 